\title{
Large area photon detectors for large-scale neutrino physics experiments: single large area PMTs and multi small PMTs.
}

\section{S. Lubsandorzhiev $a^{*}$}

a Institute for Nuclear Research of the Russian Academy of Sciences, Moscow, Russia

E-mail: sultimeinbox.ru

More than 40 years ago beginning of works on deep underwater high energy neutrino telescope projects (DUMAND and Baikal) inspired development of new photon detectors: large area photomultipliers (PMTs), multi small PMT optical modules, small PMTs equipped with wavelength shifting plates and rods and even small area solid state photon detectors for such kind application. Now days we witness rebirth of the multi small PMT approach and it started to compete quite successfully with a single large area photon detector approach. The latter have been reigning supreme for almost half century. But recent developments of astroparticle physics experiments demonstrated good competiveness of the "multi small PMTs" idea. Several projects of astroparticle physics experiments may serve as good examples, Km3NET project and coming JUNO experiment among them. We present pros and cons of both approaches.

37th International Cosmic Ray Conference - ICRC2021

12-23 July, 2021

Berlin, Germany 


\section{Introduction}

Vacuum photoelectron multipliers are the most widely used photodetectors in large-scale experiments in high energy physics and astroparticle physics, see [1] and all references therein. They play particular role in neutrino physics. Practically in all neutrino experiments vacuum photomultipliers are basic detecting elements. Presently an overwhelming number of large-scale neutrino experiments exploit large area photomultipliers. Despite the fact that for the last ten years possibilities to use a big number of small photomultipliers instead of a single large area photomultiplier are actively discussed, like in KM3NeT/ORCA [2] and PINGU [3], vacuum photomultipliers with large sensitive area are still the most demanded photodetectors for largescale neutrino experiments.

Getting rapid development for the last two decades silicon photomultipliers (SiPM) can not presently compete seriously for such kind of applications, first of all, due to their miniature sizes and high level of dark current counting rate.

Recently to get high energy resolution a double calorimetry approach is being developed like in JUNO experiment $[4,5]$. In this approach small 3-inch photomultipliers are used along with large area 20-inch photomultipliers. The central detector of the JUNO experiment is viewed by approximately 18 thousands 20 -inch photomultipliers and 25 thousands small 3 -inch photomultipliers.

Presently high sensitive large area photomultipliers with hemispherical photocathodes not less than $20 \mathrm{~cm}(8 \mathrm{inch})$ in diameter are produced by only four manufacturers around the world - Hamamatsu Photonics (Japan), ET Enterprises Ltd. (UK), NNVT (China) and HZC Photonics (China).

Of course, $20 \mathrm{~cm}$ size border for large area photomultipliers is just a convention accepted by a community. In the following sections we will review existing large area photomultipliers so far.

\section{8-inch photomultipliers.}

1. R5912 series of PMTs.

This series has a famous predecessor - R1408, also 8-inch PMT which was used in a number of great neutrino experiments like IMB (IBM-3) [6] (registration of neutrino signal form supernovae SN1987A), MACRO [7], and SNO [8]. It is interesting that old R1408 PMTs from $\mathrm{SNO}$ are being planned to be used still in $\mathrm{SNO}+$ experiment [9].

R5912 was used in MILAGRO [10] and Daya Bay [11]. Now it is used in HAWC [12] and Super-Kamiokande (in muon veto part) [13], TAIGA experiment [14], LHAASO experiment [15] etc.

This PMT has $19 \mathrm{~cm}$ in diameter photocathode effective area. Photocathode (bilakali $\mathrm{K}_{2} \mathrm{CsSb}$ ) sensitivity region spans from $300 \mathrm{~nm}$ to $650 \mathrm{~nm}$. Maximum of photocathode sensitivity is at $370-80 \mathrm{~nm}$ where its value reaches $28-30 \%$. Mean value of photocathode quantum efficiency is $\sim 25 \%$. A modification of this PMT R5912-100 is equipped with super bialkali 
photocathode with quantum efficiency at sensitivity maximum is more than $35 \%$. There are samples of this PMT with quantum efficiency exceeding $40 \%$.

\section{ETL9350 series produced by ET Enterprises.}

PMTs from this series were used and still being used in such famous experiments like MACRO, SNO, Borexino [16], GERDA [17], Tunka-133 [18], Neutrino-4 [19] etc.

Effective area of bilakali photocathode has diameter of $22 \mathrm{~cm}$. Sensitivity region is 300$650 \mathrm{~nm}$. This series of PMTs exploits two types of bialkali photocathodes $-\mathrm{K}_{2} \mathrm{CsSb}$ and $\mathrm{Rb}_{2} \mathrm{CsSb}$. The latter has more sensitivity in green region in comparison with the former one. So, sometimes the $\mathrm{Rb}_{2} \mathrm{CsSb}$ photocathode is called like "green-extended" photocathode. Maximum of photocathode sensitivity reaching at $360-380 \mathrm{~nm}$ is $28 \%$, but direct measurements show usually lower value no more than $25 \%$.

3. There is a new development of Hamamatsu 8-inch PMT - R14688-100 with less than $1 \mathrm{~ns}$ (FWHM) time resolution (TTS) [40].

\section{10-inch photomultipliers}

Photomultipliers of this size are presented by only one type of PMTs - series of R7081. This PMT became a real "working horse" of contemporary large-scale neutrino physics experiments. It is used in a great variety experiments in cosmic ray physics and ground based gamma-astronomy (Ice-TOP [20], TAIGA-HiSCORE [21], etc), reactor neutrino experiments (Double Chooz [22], RENO [23], STEREO [24], etc.) and neutrino telescopes (IceCUBE [25], ANTARES [26], GVD [27], etc.), new liquid scintillator experiments like LBNT (Large Baksan Neutrino Telescope) [28]. The PMT has a glass bulb with a specially designed shape to get more uniform time and amplitude response. So, for that reason the working area of photocathode is noticeably less than the outer size of glass bulb $22 \mathrm{~cm}$ or even less and $25 \mathrm{~cm}$ correspondingly. The series includes PMTs with ordinary bialkali photocathode (R7081, R7081-10 and R708120) with maximum quantum efficiency of $25-30 \%$ and super bilakali one (R7081-100) with maximum quantum efficiency of more than 35\%. There are samples of R7081-100 with quantum efficiency more than $40 \%$.

\section{20-inch photomultipliers}

1. R12860 series of PMTs produced by Hamamatsu Photonics. This series has its famous predecessors R1449 and R3600 used in KAMIOKANDE [29] and Super-KAMIOKANDE [30] experiments respectively. R7250 17-inch PMT used successfully in KamLAND [31] experiment relates also directly to those series of PMTs because it utilizes the same 20-inch glass bulb. It should be noted that R7250 PMT continue to be successfully used in KamLAND-Zen [32] experiment.

The PMTs of these series are equipped with conventional bilakali phptocathode. The effective area of photocathode is $43-46 \mathrm{~cm}$ and $38 \mathrm{~cm}$ for R12860 and R7250 respectively. Quantum efficiency maximum of $25-30 \%$ is reached at 360-400 nm. In contrary to R1449 and R3600 series exploiting obsolete venetian-blind dynode system, R12860 PMTs were undergone a deep modernization of dynode system and electron optics to get higher collection and photon 
detection efficiencies. The glass envelope of PMTs was modified too mostly increasing curvatures radii to get more resistive to hydrostatic pressure.

5000 R12860 PMTs will be used in JUNO experiment. This PMT is a good candidate for use in Hyper-KAMIOKANDE [33] experiment.

2. Large area MCP-PMT.

This MCP-PMT is a large area hybrid photomultiplier using micro-channel plates for photoelectron multiplication. It has a bilakali high quantum efficiency hemispherical photocathode $50 \mathrm{~cm}$ in diameter. Maximum of quantum efficiency is more than $33-35 \%$ and reached at 380-400 nm. 18000 MCP-PMTs will be used in JUNO experiment $[4,5]$.

\section{9-inch, 11-inch and 12-inch photomultipliers}

There are very interesting developments of 11-inch (R11780, Hamamatsu) and 12-inch (ETL D784UKLFB, ET Enterprises) photomultipliers. Their glass bulbs were developed to withstand higher hydrostatic pressure up to 11-12 atm. ETL D784UKLFB PMT is available now in its low gain version ET9352K. This PMT was already used in pilot samples of optical modules of TAIGA-HiSCORE [21] experiment.

9-inch XP1805 and 11-inch XP1807 were developed by Photonis, France in 2000s. Later their production was revived by HZC Photonics in China.

\section{Small size 3-inch photomultipliers}

Relatively recently it has been getting more and more popular an approach to replace one single large area photomultiplier by many small area photomultipliers. In this approach one optical module consists of several dozens of 3-inch photomultipliers. Presently it is so called "Multi-PMTs OM" [35] approach. It should be noted that the approach was discussed actively in the 1970s during first deep underwater neutrino telescope development (see [36, 37] and all references therein). Esso Flickt revived this approach in 2001 [36, 37].

This approach has undoubtedly its advantages and disadvantages. There are a number of candidates of 3-inch photomultipliers to be used for such kind of application - R14374 produced by Hamamatsu Photonics, XP72B22 from HZC Photonics and "PMT-Sphere" developed by MELZ FEU among them. Using many small PMTs instead of a single large area PMT is based on proposition that repetitiveness of small PMTs parameters in mass production is as high as in semiconductor industry. Unfortunately it is not the case with mass production of vacuum photomultipliers where share of hand work is still rather high. Moreover, so far there are no proves of a longterm stability and robustness of "multi small PMTs" approach.

On the other hand there is a "double calorimetry" strategy being implemented in JUNO experiment $[4,5]$ where a great number ( 25 thousands) of 3-inch PMTs will be used along with large area 20-inch PMTs in order to get high energy resolution of the whole experiment. 


\section{Large Area Picosecond Photodetector}

For the last decade a LAPPD (Large Area Picosecond Photodetector) [38] was developed. LAPPD is a hybrid phototube with flat photocathode and MCP as photoelectron multiplier. LAPPD utilizes proximity focusing system allowing very fast time resolution and timing. So far the largest area reached is $350 \mathrm{~cm}^{2}$ [39]. Flat bialkali photocathode has maximum sensitivity $\sim 25 \%$. The idea is to cover large area by LAPPDs. But, to use LAPPDs in large-scale astroparticle physics experiments, in deep underwater neutrino telescopes in particular, is rather dubious due mostly to flat geometry, with technological problems to produce high sensitive photocathodes in such a geometry etc.

\section{Conclusion.}

We reviewed large and small area photomultipliers used or to be used in large-scale experiments in astroparticle physics. One can conclude that it is quite possible that large and small area photomultipliers will go together hand in hand forming a kind of "symbiosis" for sake of higher sensitive large-scale experiments of the next generation in astroparticle physics, in neutrino physics in particular

\section{Acknowledgements.}

The author is very grateful to Dr. V.Ch. Lubsandorzhieva for careful reading of the manuscript and many invaluable remarks.

\section{References}

[1] B.K.Lubsandorzhiev. Evolution of Ideas in Photon Detection. Physics of Particles and Nuclei. 2016. Vol.47. No.6. P.957-967.

[2] P. Kooijman et al. (KM3NeT Collaboration). Multi-PMT Optical Module. Nucl. Instrum. and Meth. A. 2006. Vol. 567. P. 508.

[3] Jason Koskinen. Atmospheric neutrino results from IceCube-DeepCore and plans for PINGU // J. of Phys: Conf. Ser. 2017. Vol. 888

[4] Fengpeng An, et al. Neutrino Physics with JUNO. Journal of Physics G: Nuclear and Particle Physics. 43 (2016) 030401

[5] A. Abusleme et al. JUNO Physics and Detector, arXiv:2104.02565.

[6] R. Becker-Szendy et al., Nucl. Instrum. and Meth. A. 1993. Vol. 324. P. 363.

[7] M.Calicchio et al., THE MACRO DETECTOR AT THE GRAN SASSO LABORATORY Nucl. Instr. Meth. Phys. Res. A264(1988)18.

[8] Smith N.J.T. The SNOLAB deep underground facility // The European Physical Journal Plus. 2012. Vol. 127, № 9.

[9] Biller S. SNO+ with Tellurium, Physics Procedia. 2015. Vol. 61. 
[10] Atkins, R., et al., The Astrophysical Journal, 608 (2004) 680-685

[11] An F.P. et al. Observation of Electron-Antineutrino Disappearance at Daya Bay // PRL. 2012. Vol. 108, № 17

[12] A.U. Abeysekara et al., Nucl. Instrum. and Methods. A. 2018. V. 888. P.138.

[13] Fukuda S., et al. The Super-Kamiokande detector, Nucl. Instrum. and Methods. 2003. V. A501. P.418.

[14] N.M. Budnev, et. al. Nucl. Instrum. and Meth. A, 2020. V.958. 162113.

[15] Z. Cao, Chin. Phys. C, 34 (2)(2010): 249-252

[16] Bellini G. et al. Final results of Borexino Phase-I on low-energy solar neutrino spectroscopy, Physical Review D. 2014. Vol. 89, № 11. P. 112007.

[17] K. Freund, et al. The Performance of the Muon Veto of the Gerda Experiment, EPJC 76:298 2016.

[18] Antokhonov B.A., et al. A new $1 \mathrm{~km}^{2}$ EAS Cherenkov Array in the Tunka Valley, Nucl. Instrum. and Meth. A. 2011. V.639. P.42-45.

[19] Serebrov A.P. et al. First Observation of the Oscillation Effect in the Neutrino-4 Experiment on the Search for the Sterile Neutrino, JETP Letters. 2019. Vol. 109, № 4.

[20] X. Bai et al., Nucl. Phys. B Proc. Suppl. 2008. V.175-176. P.415.

[21] M. Kunnas, et al. Nucl. Instrum. and Meth. A. 2014. V.742. P.269.

[22] Abe Y. et al. Reactor ve disappearance in the Double Chooz experiment // Physical Review D. 2012. Vol. 86, № 5.

[23] Ahn J.K. et al. Observation of Reactor Electron Antineutrinos Disappearance in the RENO Experiment, Physical Review Letters. 2012. Vol. 108, № 19. P. 191802.

[24] N. Allemandou et al. JINST 13, 07(2018):P07009

[25] M.G. Aarsten et al. JINST 12(2017)P03012

[26] M.Circella. The construction of ANTARES, the first undersea neutrino telescope // Nucl. Instrum. and Meth. A. 2009. V.602. P.1.

[27] A.D. Avrorin et al. arXiv:1808.10353.

[28] N.A. Ushakov, et al. New large-volume detector at the Baksan neutrino observatory of the INR RAS: Detector prototype, J. Phys.: Conf. Ser. 1787(2021)012037.

[29] T. Kajita, M. Koshiba, A. Suzuki. On the origin of the Kamiokande experiment and neutrino astrophysics // EPJH. 37(2012)33.

[30] S. Fukuda et al. Nucl. Instrum. and Meth. A. 2003. V.501. P.418.

[31] Eguchi K. et al. First Results from KamLAND: Evidence for Reactor Antineutrino Disappearance, Physical Review Letters. 2003. Vol. 90, № 2.

[32] A. Gando et al., PRL 117(2016)082503.

[33] di Lodovico F. et al. J. of Phys.: Conf. Ser. 2017. Vol. 888. P. 012020.

[34] T. Adam, et al. JUNO conceptual design report. arXiv:1508.07166. 
[35] P. Kooijman et al. (KM3NeT Collaboration). Multi-PMT Optical Module, Nuclear Instruments and Methods A. 2006. V.567. P.508.

[36] B.K.Lubsandorzhiev. Evolution of Ideas in Photon Detection, Physics of Particles and Nuclei. 2016. Vol.47. No.6. P.957-967.

[37] Bayarto Lubsandorzhiev. "New" and "Old" ideas in photon detection, PoS (PhotoDet2015)040.

[38] Adams T.W. et al. A brief history of the large-area picoseconds photodetectors, arXiv:1603.01843.

[39] M.J. Mino et al. Large area picoseconds photodetector $\left(L A P P D^{T M}\right)$ offers fast timing for nuclear physics and medical imaging, IL NUOVO CHIMENTO 43 C (2020) 11.

[40] https://www.hamamatsu.com/eu/en/product/type/R14688-100/index.html 